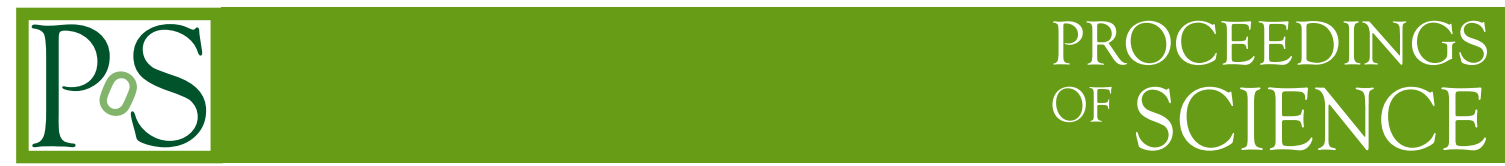

\title{
Witten parameter in the SU(2)-gluodynamics
}

\author{
Vladimir Goy* and Alexander Molochkov \\ Far Eastern Federal University \\ E-mail: vovagoy@gmail.com, molochkov.alexander@gmail.com
}

\begin{abstract}
The purpose of the article is to study the surface operator in an $S U(2)$ non-Abelian gauge field theory. We analyse abelian projection of the $S U(2)$ symmetry to the $U(1)$ group calculating the Witten parameter by the lattice method. We have used multilevel and multi-hit algorithms for the sake of statistical confidence. We have demonstrated that the Witten parameter depends on the surface area and volume in both phases. Consequently, the Witten parameter cannot be considered as the order parameter of confinement-deconfinement phase transition.
\end{abstract}

XXI International Baldin Seminar on High Energy Physics Problems September 10-15, 2012

JINR, Dubna, Russia

*Speaker. 


\section{Introduction}

In the four-dimensional gauge filed theory the most important probes for the phase states are the Wilson and t'Hooft line operatros that are difined on one-dimensional curves in the space-time. For example, these line-operators define order parameters for the confinemnet-deconfiment phase transition of the QCD vacuum. However, for more detail understanding of four-dimensional gauge field theory dynamics we need additional probes expressed by operators defined on the subspaces with higher dimensions. Possible candidates are operators that are defined on the two-dimensional surface in the four-dimensional space-time.

As an example we consider applications of the surface operator to study of the phase properties of QCD. One of the possible explanations of the quark confinement is the condensation of magnetic monopoles in the vacuum [1] as dual superconductor models. The BCS theory of superconductivity explains superconductivity as the result of the condensation of electric chargers to cooper pairs. In this case a string occurs between monopoles. In a dual superconductor an analogous effect occurs through the condensation of magnetic charges (also called magnetic monopoles), and a string connects electrically charged particles. According to 't Hooft [2], monopoles can appear as a result of partial break of gauge symmetry. In this work we break $S U(2)$ symmetry saving $U(1)$ group symmetry.

A surface operator [3] is sensitive to existence of monopoles. It was first introduced by Witten $[4,5]$. It is determined by the divergence of the chromomagnetic field through a closed surface. We use the lattice calculations to study the structure of the vacuum in $S U(2)$ gluodynamics.

In non-abelian pure gauge theories, the expectation values of large surface operators are difficult to compute via numerical simulation because the increase of surface area makes the signalto-noise ratio decay very fast. We adapt the multilevel scheme [6] introduced for line operators to work with surface operators when area exceeds $1 \mathrm{fm}^{2}$.

\section{Witten parameter}

In the trivial vacuum, for example, in electrodynamics, the vector flow of magnetic field through a closed surface is identical to zero:

$$
\oint \mathbf{H} \cdot d \mathbf{S} \equiv 0
$$

In the lattice calculation we use a phase like $e^{l \varphi}$, therefore the identity (2.1) acquires the following form:

$$
e^{\imath \kappa \oint \mathbf{H} \cdot d \mathbf{S}} \equiv 1,
$$

where $\kappa$ is a dimensional coefficient. In Abelian theories this identity works in a simply connected space. If the space topology is non-trivial or a group symmetry is non-Abelian the identity (2.2) does not necessarily works. Hence, in lattice quantum chromodynamics we have:

$$
e^{\imath \kappa \sum \mathbf{H}_{k} \cdot \Delta \mathbf{S}_{k}} \neq 1
$$

where $\mathbf{H}_{k}$ is the magnetic field vector on the lattice plaquette with index $k, \Delta \mathbf{S}_{k}$ is a surface area of the plaquette (with the normal vector in the center of the plaquette), and the integral is calculated over a closed surface made up of lattice plaquettes. 
Thus, we consider the following value to be the Witten parameter:

$$
W_{p}(S)=R e \prod_{S} e^{\imath \theta_{p}}
$$

where $\theta_{p}$ is a plaquette angle. Essentially, the plaquette angle is a quantitative measure of the gauge field impact on an external source moving along the contour of a plaquette. This angle relates to magnetic field flow through the plaquette surface:

$$
\kappa \int_{S} \mathbf{H} \cdot d \mathbf{S}=\kappa \oint_{C} \mathbf{A} \cdot d \mathbf{l}=\theta_{p},
$$

where integration over $d \mathbf{l}$ carried out on a path covering the surface $S$.

Let's rewrite the magnetic field flow:

$$
\int_{S} \mathbf{H} \cdot d \mathbf{S}=\int_{S} F_{i k} d \sigma_{i k},
$$

where $F_{i k}$ is the gauge field tensor, $d \sigma_{i k}$ is a surface element (we do not differ between upper and lower indices because all the calculations are performed in the Euclidean space-time after the Wick rotation), and $i, k=1,2,3$ is the space direction. In this work we consider the pure gauge field theory with $S U(2)$ group symmetry. Thus, the $\theta_{p}$ relates to the $F_{\mu \nu}$ by the following formula:

$$
F_{p}=\widehat{1} \cos \theta_{p}+\imath n_{i} \sigma_{i} \sin \theta_{p}
$$

where $n_{i}$ is a vector on the unit sphere, $\sigma_{i}$ is the Pauli matrices, $F_{p}$ is a value of the gauge field tensor $F_{\mu v}$ on the plaquette. Then, for the $\theta_{p}$ we can write the following definition:

$$
\theta_{p}=\arccos \left(\frac{1}{2} \operatorname{Tr} F_{p}\right)
$$

All the phases are calculated on the surface of a cube in the four-dimensional space-time. The range of function $\arccos (x)$ is $[0, \pi]$. In the gauge group $U(1)$ the range of the angle is $[0,2 \pi]$. Hence, on one side of the cube the phase is selected as $+\arccos \left(\frac{1}{2} \operatorname{Tr} F_{p}\right)$, whereas on the opposite side it is $-\arccos \left(\frac{1}{2} \operatorname{Tr} F_{p}\right)$.

The Witten parameter is related not only to the chromomagnetic field, but also to the correlation function of an average plaquette which is defined as follows:

$$
C(l)=\left\langle\left(1-\frac{1}{2} \operatorname{Tr} F_{p}(x)\right)\left(1-\frac{1}{2} \operatorname{Tr} F_{p}(x+l)\right)\right\rangle_{x}
$$

where $F_{p}$ is the plaquette variable defined by $F_{p}=U_{i j} U_{j k} U_{k l} U_{l i}$, where $U_{i j}, U_{j k}, U_{k l}, U_{l i}$ are plaquette link variables. The bigger surface we take, the smaller gets the correlation between plaquettes located on the opposite planes of the cube. Thus, the phase $\theta_{p}$ has the same correlation function which in turn influences the Witten parameter. 


\section{Witten parameter on the lattice}

The partition function has the following formula:

$$
Z=\int(d U) e^{-S(U)}
$$

We use the Wilson formalism of the lattice theory [7]. The action for $S U(2)$ theory can be written as $S(U)=\beta \sum_{p}\left(1-\frac{1}{2} \operatorname{Re} \operatorname{Tr} F_{p}\right)$, where $\beta=4 / g_{0}^{2}$ and $g_{0}$ is the gauge coupling constant. We can calculate any observed value of a physical quantity $A$ by the next formula:

$$
\langle A\rangle=Z^{-1} \int(d U) A(U) e^{-S(U)},
$$

where $A(U)$ is a physical quantity calculated on the lattice configuration $U$ and the integration is taken over all configurations with the weight equal to $e^{-S(U)}$.

In this approach we need to generate some quantity lattice configuration with the weight $e^{-S(U)}$. This problem is solved with the use of Monte Carlo algorithms [8]. Further we calculate the observed physical quantity on these configurations and average them. To generate the configuration we use cold start, cyclic boundary conditions and other parameters shown in Table 1 . To calculate errors we use $99 \%$ confidence interval, therefore for 50 configurations errors are calculated as $2.8 \sigma$, where $\sigma$ is the typical dispersion.

To study the Witten parameter we prepared a set of configurations in both phases. We calculate a Polyakov loop to verify the phase state on the configurations. The loop is defined as follows

$$
L(T)=\frac{1}{2} \operatorname{Tr} \exp \left(l_{0} \int_{0}^{1 / T} A_{0} d t\right),
$$

where $t$ is a cyclic variable with period $1 / T, T$ is the temperature on the lattice. The Polyakov loop is the order parameter of confinement-deconfinement phase transition. In the confinement phase it equals zero, and in the deconfinement phase it is different from zero. In the Table 2 we show lattice characteristic used in the calculations. On the lattice the Polyakov loop is a Wilson line composed of lattice links in the temporal direction and closed with the periodic boundary condition. The Polyakov loop on the lattice is defined by the following formula:

$$
L(\mathbf{x})=\frac{1}{2} \operatorname{Tr} \prod_{t=0}^{N_{t}-1} U_{0}(t, \mathbf{x}),
$$

where $U_{0}(t, \mathbf{x})$ is the time direction link.

Table 1: Monte Carlo parameters.

\begin{tabular}{|c|c|}
\hline Thermalization iteration & 2000 \\
\hline Correlation iteration & 200 \\
\hline Quantity configurations & 50 \\
\hline
\end{tabular}


Table 2: Lattices used to search the volume dependence.

\begin{tabular}{|c|c|c|c|}
\hline Phase & Size lattice & $\beta$ & $L(T)$ \\
\hline Deconfinement & $4 * 30^{3}$ & 2.55 & $0.349 \pm 0.002$ \\
\hline Confinement & $41^{4}$ & 2.55 & $0.0002 \pm 0.0006$ \\
\hline
\end{tabular}
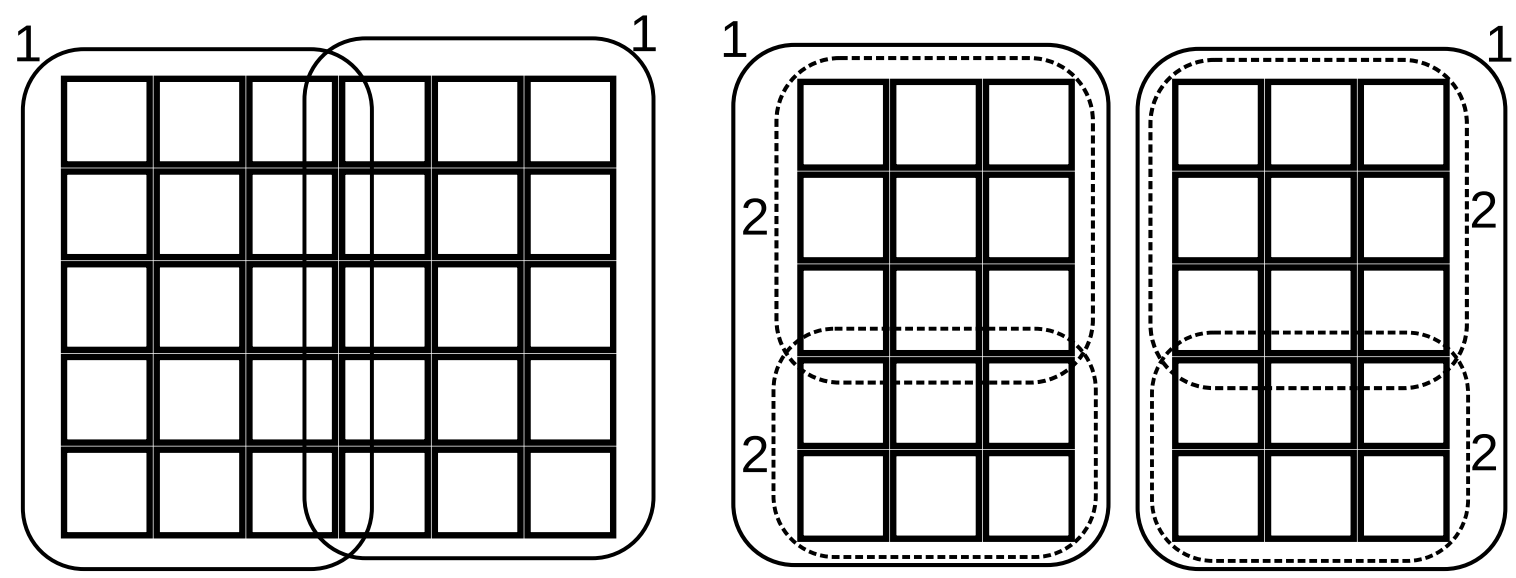

Figure 1: The plane is divided into two pieces on each level of recursion. 1 shows that the phase on the surface (surrounded by black solid line) is calculated on the first level of recursion, and 2 is computed on the second level of recursion.

In the lattice approach we select a cube in the $3 \mathrm{~d}$ space (the lattice lives in the four-dimensional space-time) with some length of the edge on the lattice. The phase is calculated on each plaquette on the surface of the cube, the result is obtained by composition of these phases. Next we calculate the Witten parameter at different points in the lattice configuration and average them. The final result is obtained by averaging on the set of configurations.

We consider cubes with edge length ranging from $1 a$ to $13 a$ ( $a$ is the lattice scale) and surface area value ranging from 6 to 1014 plaquettes, respectively. For the sake of statistical confidence we use multilevel [6], multi-hit [9] algorithms and MPI parallelism for fast calculations.

\subsection{Multilevel}

We can fractionize the cube into 6 planes. Thus, we need to learn how to calculate the phase on this plane. We use multilevel scheme to write a recursive function which makes the following:

1. If the depth of recursion exceeds some parameter depth $h_{\max }$ or the current plane contains only one plaquette, then the phase is calculated by the multi-hit algorithm and the function returns this value of the phase.

2. The current plane is divided into two pieces along the axis where length of the plane edge is maximum, see Figure 1. 
3. This function is called recursively for each piece of the plane and calculates the phase on this plane $\varphi$ which is the sum of phases on individual pieces of the plane. The phase $\varphi$ is appended to an array $\left\{\varphi_{i}\right\}$, where $i=1 \ldots N_{\varphi}, N_{\varphi}$ is the constant.

4. When the amount of elements $\varphi$ in the array $\left\{\varphi_{i}\right\}$ is equal to the number $N_{\varphi}$ then the function returns $\operatorname{Arg}\left(N_{\varphi}^{-1} \sum_{i} \cos \varphi_{i}+\imath N_{\varphi}^{-1} \sum_{i} \sin \varphi_{i}\right)$. If the amount is less than $N_{\varphi}$ we perform a few Monte Carlo runs on the current plane and turn back to step 3.

Then we test this function. For this purpose we select different values of depth, ranging from 1 to 3 , and calculate the dependence of the Witten parameter on a surface area on some set of lattice configurations. The multilevel algorithm applied to calculate the surface operators appears to have very good convergence behaviour. The results of calculations for recursive depth equal to two and three are close. The value of depth equals three in all calculations in this paper.

\subsection{Multi-hit}

The main idea of the multi-hit algorithm is that the phase on the plaquette is defined by bound conditions. We can substitute the phase calculated on the plaquette to the phase expressed by bound links. We cannot do it analytically, but we can use Monte Carlo algorithm to obtain more accurate phase value. For this purpose we calculate a set of phases on one plaquette. Between calculations we do a few Monte Carlo runs on the plaquette. After that, the final phase can be obtained by averaging the set of phases.

These two algorithms give the best accuracy in the calculations of the Witten parameter. However, computing time increases. Therefore, we use MPI in our calculations.

\section{Results}

All calculations are performed on 50 configurations in 1000 points on each lattice configuration. For both phases results are shown at the Figure 2. We can say that these two dependencies are the same. To understand better the behaviour of the Witten parameter we fit our dependence as follows

$$
W_{p}(S, V)=e^{-\sigma S-\gamma V},
$$

where $\sigma$ is a surface coefficient, $\gamma$ is a volume coefficient, $S$ is a surface area, $V$ is the cube volume. We use minuit 2 library from ROOT package [10] to fit numerical results. The Figure 2 shows that the fitting is good if $\sigma, \gamma \neq 0$. Then in both phases the Witten parameter depends on the cube volume and surface area.

If we look at the dependence on $\beta$ we see that the parameter vanishes in the continuum limit. See Figure 3. Clearly, the vacuum expectation value is suppressed by the ultraviolet divergence of self-energy which is proportional to the closed surface area. It means that we have the divergence of the surface coefficient $\sigma=\sigma(a) \stackrel{a \rightarrow 0}{\longrightarrow} \infty$, where $a$ is the lattice scale. The scale in the continuum limit must tend to zero. The divergence corresponds to self- energy of coloured dipoles on the 

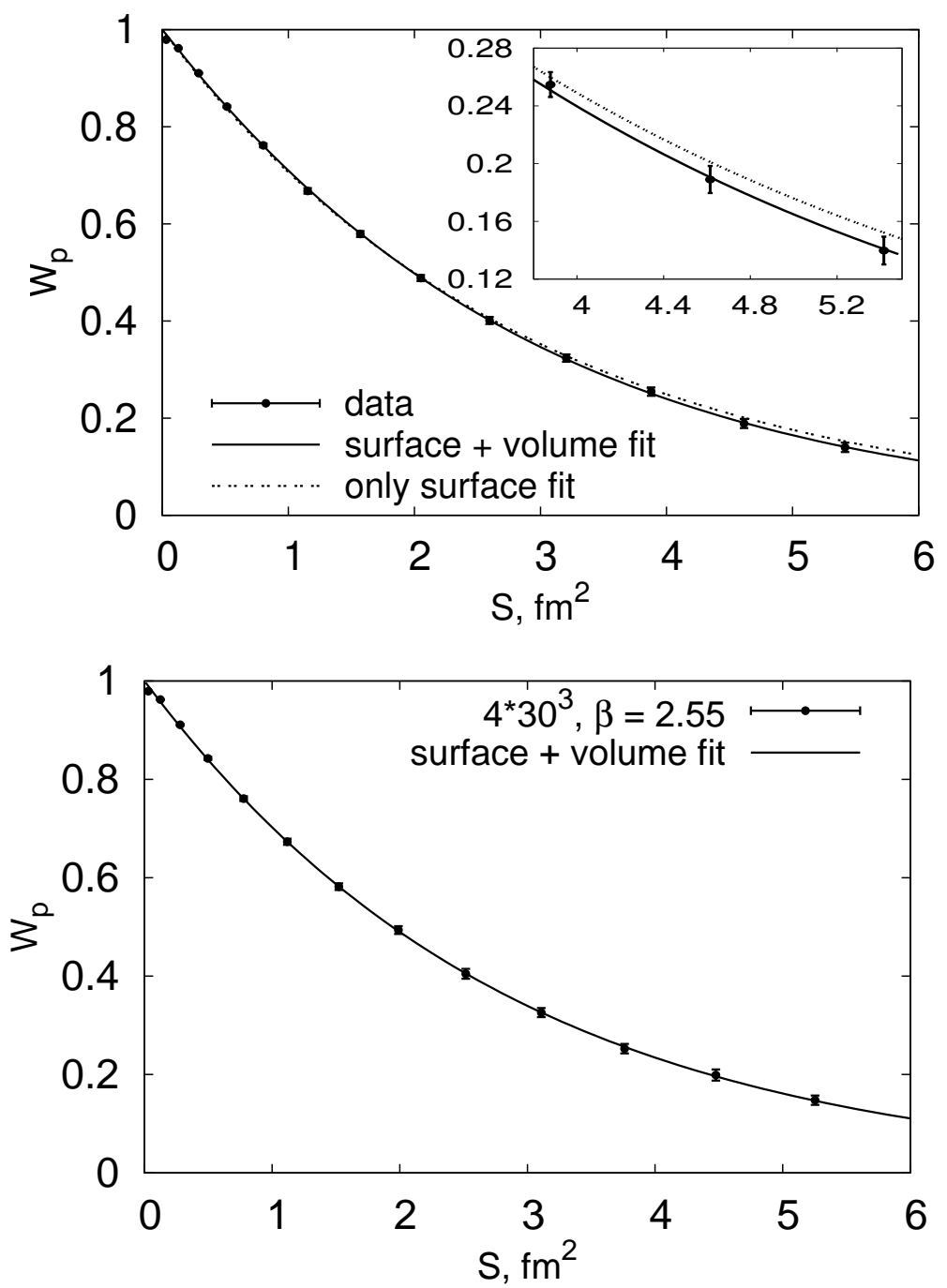

Figure 2: Dependence of the Witten parameter on a surface area in the confinement phase (up panel) and comparison of fittings. Lattice size is $41^{4}$ and $\beta=2.55$. The same can be observed in the deconfinement phase (bottom panel).
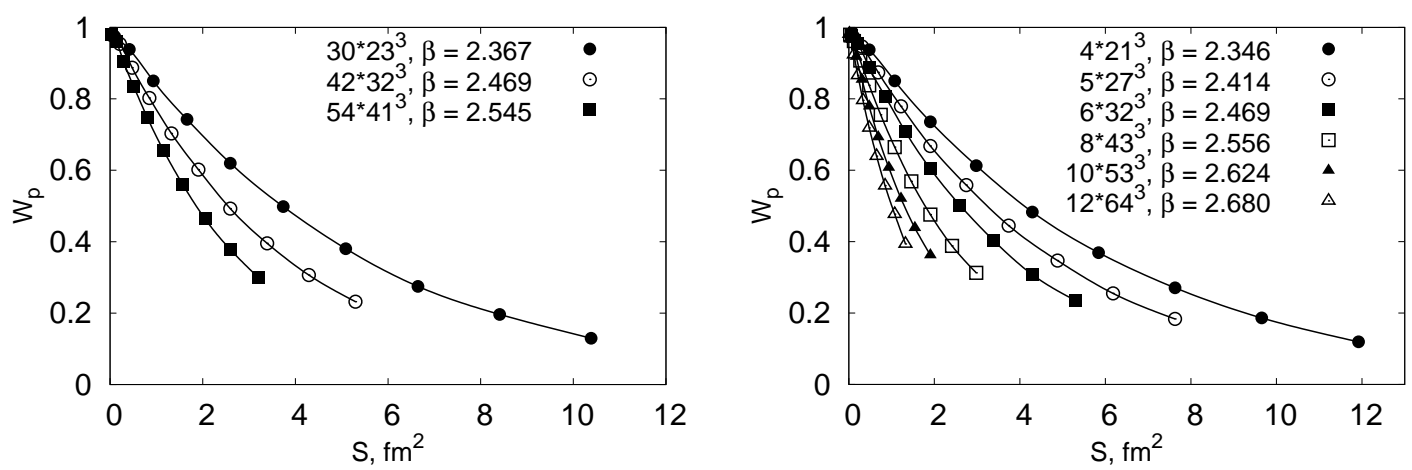

Figure 3: The dependence of the Witten parameter with different $\beta$. In the confinement phase - the left panel. In the deconfinement phase - the right panel. 


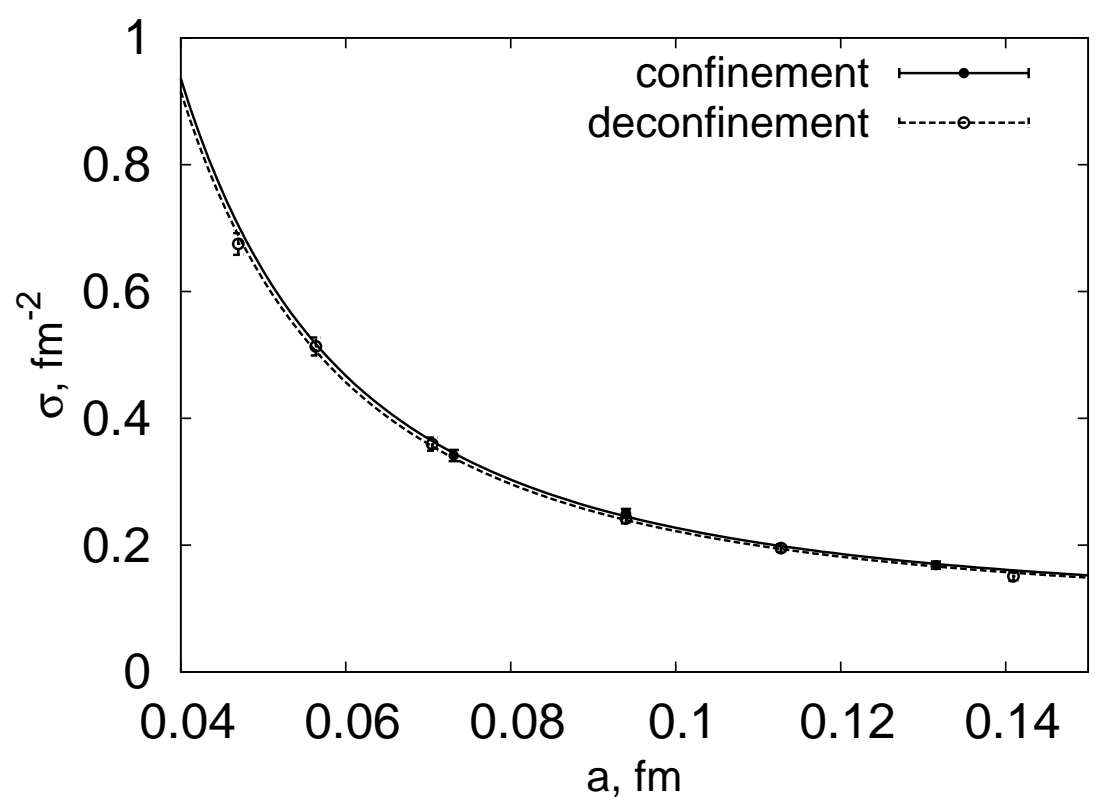

Figure 4: Dependence of the $\sigma$ on the lattice spacing $a$ in both phases.

surface. It is analogous to divergence in case of Wilson lines:

$$
\left\langle\operatorname{Tr} P \exp \left\{-\int_{C}^{\wedge} \hat{A}_{\mu} d x_{\mu}\right\}\right\rangle \sim \exp \left\{- \text { const }^{2} L / a\right\},
$$

where $L$ is the perimeter of the Wilson line $C, a$ is the lattice spacing, $g^{2}$ is a coupling constant, and we keep only the most divergent piece. A description of magnetic degrees of freedom and surface operators may be found in Ref. [11].

The Figure 4 shows how $\sigma$ depends on scale $a$. Only $\sigma$ diverges in the continuum limit. The volume coefficient $\gamma$ does not depend on lattice scale. According to the formula (4.2) we can suppose that surface divergence has the following form

$$
\sigma(a)=\sigma_{p h}+\sigma_{d i v} / a^{2}
$$

where $\sigma_{p h}$ is the physical coefficient and $\sigma_{d i v}$ is the divergence coefficient. After fitting we obtain $\sigma_{p h}=(0.091 \pm 0.007) \mathrm{fm}^{-2}$, or $(3.6 \pm 0.3) * 10^{3} \mathrm{MeV}^{2}$. This approximation is illustrated in Figure 4.

One can say that the Witten parameter depends on a surface area and volume in both phases. Consequently, the Witten parameter cannot be considered as the order parameter of confinementdeconfinement phase transition. This result is similar to the behaviour of spatial Wilson loops expectation value as the spatial Wilson loop also does not sense the phase transition. It might be interesting to study surface operators to calculate the Witten parameter on the cube with two spatial axis and one temporal axis. In this case the parameter should sense the phase transition. 


\section{Acknowledgments}

We are thankful to Dr. M. I. Polikarpov, Dr. P. V. Buividovich, Dr. V. G. Bornyakov, Dr. V. I. Zakharov and Dr. V. V. Braguta for interesting and useful discussions. The authors are much obliged to Dr. A. M. Shirokov for useful comments. Many thanks to Miss Nastasiy for the help with English. The work was supported by the Russian Ministry of Science and Education by the federal program "Kadry", Agreement No. 8174. Numerical calculations were performed with the equipment of the Center for Collective Use "Far Eastern Computing Resource" (Vladivostok).

\section{References}

[1] M. N. Chernodub and M. I. Polikarpov, arXiv:hep-th/9710205 [hep-th] (1997).

[2] G. 't Hooft, Nuclear Physics B 190, 455 (1981).

[3] S. Gukov, "Phases of gauge theories and surface operators" the talk presented at the workshop "Prestrings", 11-15 August, 2008, Zürich, Switzerland.

[4] S. Gukov and E. Witten, arXiv:hep-th/0612073v2 [hep-th] (2007).

[5] S. Gukov and E. Witten, arXiv:0804.1561 [hep-th] (2008).

[6] M. Lüscher and P. Weisz, JHEP 0109, 010 (2001), arXiv:hep-lat/0108014 [hep-lat].

[7] K. Wilson, Phys. Rev. D 10, 2445 (1974).

[8] M. Creutz, Quarks, gluons and lattice Cambridge University Press, Cambridge, 1983.

[9] G. Parisi, R. Petronzio and F. Rapuano, Physics Letters B 128, 418 (1983).

[10] The ROOT library, http://root.cern.ch/drupal/.

[11] A. Di Giacomo and V. I. Zakharov, Phys. Atom. Nucl. 73, 711 (2010), arXiv:0806:2938 [hep-th]. 